\title{
Tetrads and BF-gravity
}

\section{Shishanin A.O.}

Bauman Moscow State Technical University, Moscow, Russia;

E-mail: Shishanin <shishandr@rambler.ru>;

It is provided an overview of the tetradic approach (the Palatini formalism) for gravity. It is considered some theory with new additional field B. It is shown that this model is equivalent the Einstein gravity with cosmological term and topological term in the tetradic approach. It is considered the limit of the gravitational constant $\mathrm{G}$ goes to 0 . In the leading non-zero order of $\mathrm{G}$ is obtained some equation for spin connection which is equation of zero condition. It is discussed relation of this equation with MacDowell-Mansouri-Stelle-West gravity.

Keywords: tetrads, spin connections, differential forms, BF-gravity.

DOI: $10.18698 / 2309-7604-2015-1-468-472$

The Einstein gravity may be considered in the tetradic approach [1]. Naively tetrad $e$ is vector in tangent space and the metric tensor is expressed as

$$
g_{\mu v}=e_{\mu}^{a} e_{v}^{a}
$$

It is more convenient for gravity to use description of fiber bundles and differential forms. Let us consider following 1-form of tetrad $e=e_{\mu}^{a} d x^{\mu}$. The spin connection $\omega_{\mu}^{a b}$ is connection in the spinor bundle. Certainly there is the form of spin connection $\varpi=\varpi_{\mu}^{a b} d x^{\mu}$.

The tetradic approach has some advantages. At first is that here may be introduced fermions. By the way there are two different manners for fermions in general relativity. In the Palatini formalism The another dignity of the tetradic approach is more general point of view on gravity. Also tetrad $e$ is mapping between tangent fiber bundle and vector fiber bundle. If $e$ is isomorphism (the mapping of maximal rank) then the tetradic formulation of gravity is equivalent to the Einstein gravity. It seems that gravity with fermions is more delicate. Usually tetrads are used in supergravity. The Palatini action with cosmological constant $\Lambda$ in space-time manifold $\Omega$ has following form

$$
S_{P}=\frac{1}{2 \kappa} \int_{\Omega} \operatorname{tr}(F \wedge e \wedge e)-\frac{\Lambda}{\kappa} \int_{\Omega} \operatorname{tr}(e \wedge e \wedge e \wedge e) .
$$


Here $\kappa=8 \pi G$ where $G$ is the gravitational constant, $M_{P l}^{2}=1 / G$ is square of the Plank mass. $\Lambda$ is the cosmological constant, $e$ is 1 -form of tetrad and $R$ is the curvature form which can be built using 1-form spin connection $\omega$

$$
R=d \omega+\omega \wedge \omega=d \varpi+\frac{1}{2}[\omega, \varpi]
$$

Let us consider the action with some 2-form $B$

$$
S_{0}=\int_{\Omega} \operatorname{tr}(\alpha B \wedge B+(\beta R+\gamma e \wedge e) \wedge B)
$$

Then a equation of motion for $B$ is

$$
2 \alpha B+\beta R+\gamma e \wedge e=0 .
$$

Let us exclude field $B$. Then we can obtain follow action

$$
S=\frac{1}{4 \alpha} \int_{\Omega} \operatorname{tr}\left(\beta^{2} R \wedge R+2 \beta \gamma R \wedge e \wedge e+\gamma^{2} e \wedge e \wedge e \wedge e\right)
$$

Also it is possible to obtain this action using the functional integral. This action looks like action for MacDowell-Mansouri-Stelle-West gravity [2], [3], [4]. Here the first term is topological. It is proportional the second Chern class of $R$. Put $\beta=1$. There is the following system of equation

$$
\frac{\gamma}{2 \alpha}=\frac{1}{2 \kappa}, \frac{\gamma^{2}}{4 \alpha}=-\frac{\Lambda}{\kappa} .
$$

The solution of this system is

$$
\alpha=-4 \Lambda \kappa, \quad \gamma=-4 \Lambda
$$


Then the action $S_{0}$ when $G$ goes to 0 is

$$
S_{G \rightarrow 0}=\int_{\Omega} \operatorname{tr}(R \wedge B-4 \Lambda e \wedge e \wedge B)
$$

It is simply obtained the following equations

$$
d_{\varpi} B=0, \quad e \wedge B=0
$$

The following equation for $\omega$ is obtained varying this action on the field $B$

$$
d \omega+\omega \wedge \omega=4 \Lambda e \wedge e
$$

Note at first that if $\Lambda=0$ then a solution of this equation is the Minkowski space.

The simplest solution of this equation when $\Lambda \neq 0$ is $A d S_{4}$-space if $\Lambda$ is negative and $d S_{4}$ if is positive.

MMSW-gravity can be described by unified connection $A$ :

$$
A=\varpi+\frac{1}{l} e
$$

where $l$ is a constant

$$
I=\sqrt{\frac{\varepsilon}{4 \Lambda}} .
$$

Here if $\varepsilon$ is positive then $A$ is $s o(1,4)$-connection (de Sitter space). If $\varepsilon$ is negative then $A$ is $s o(2,3)$-connection (Anti de Sitter space). It can be demonstrated that the curvature form for $A$ is 


$$
F=R-4 \Lambda e \wedge e+d_{\varpi} e
$$

The last term is the torsion form. The action of MMSW-gravity has the following form

$$
S_{M M S W}=-\frac{1}{6 G \Lambda} \int_{\Omega} \operatorname{tr}(F \wedge * F) .
$$

The equation (3) is condition of zero curvature for MacDowell-Mansouri-Stelle-West gravity with zero torsion. By the way when $e$ is isomorphism and torsion is zero there is Schwarzschild-AdS-solution (AdS black hole) of the equation (3). This solution was investigated [5] due to AdS/CFT-correspondance for research of supersymmetric gauge theory at finite temperature.

Let us try to find general solution of the equation (3). Let us consider $\Lambda$ as a small parameter. Put $\omega_{0}$ flat connection. Then let us consider following expansion in power $\Lambda$

$$
\omega=\omega_{0}+\Lambda \omega_{1}+\Lambda^{2} \omega_{2}+\ldots
$$

There is the following equation for $\omega_{1}$

$$
d \omega_{1}+\left[\varpi_{0}, \varpi_{1}\right]=4 e \wedge e
$$

One can take differential $d$ by left and right sides. Then it turns

$$
d e+\omega_{0} \wedge e=0
$$

This equation means that the torsion of $\omega_{0}$ is zero. It is unclear will be the remaining terms of expansion (5) have zero torsion.

I would like to thank A.S. Losev for discussion and D. A. Polyakov for corresponding. 


\section{References}

1. Eguchi T., Peter B. G., Hanson A.J. (1980). Gravitation, gauge theories and differential geometry. Phys. rept., Vol. 66, 213.

2. MacDowell S.W., Mansouri F. (1979). Unified geometric theory of gravity and supergravity. Phys. Rev. Lett., Vol. 38, 739.

3. Stelle K.S., Peter C.W. (1980). Spontaneously broken de Sitter symmetry and the gravitational holonomy group. Phys.Rev., Vol. D 21, 1466.

4. Bekaert X., Cnockaert S., Iazeolla C., Vasiliev M.A. (2005). Nonlinear higher spin theories in various dimensions. arXiv, 0503128.

5. Witten E. (1998). Anti-de Sitter Space, Thermal Phase Transition and Confinement in Gauge Theories. Adv.Theor.Math.Phys., Vol.2, 505-532. 